\title{
Design and Implementation of Teaching and Training System Based on Radar Hardware-in-loop Simulation
}

\author{
LUO Jun \\ New Star Research \\ Institute of Applied \\ Technology \\ Hefei, China \\ Loujun08@163.com
}

\author{
ZHANG Ji-shan \\ An Hui San Lian \\ University \\ Hefei, China
}

\author{
LU Wen-jun \\ New Star Research \\ Institute of Applied \\ Technology \\ Hefei, China \\ lwj2963@sina.com
}

\author{
SUN Shuang-xi \\ Dongfeng Electrical and \\ Mechanical Technology \\ Co., Ltd. Anhui \\ Hefei, China
}

\author{
PENG Dong-hui \\ New Star Research \\ Institute of Applied \\ Technology \\ Hefei, China
}

\begin{abstract}
On the basis of a certain type of radar is proposed to establish based on $C$ / $S$ architecture, teaching and training loop simulation of radar system design. System prototype developed by the host analog receiver with the received signal, and display the data passed to the client, and then displayed by the client and the client some simulation operation. The radar system in the teaching and training practice has achieved good results.
\end{abstract}

Keywords-hardware-in-loop simulation; Client/Service mode; database

\section{SYSTEM COMPONENTS}

In reality, teaching is really kind if its cost is very expensive radar, the use of radar and the real model there is often somewhat different, in actuality, there is a lot of limitations, while the cost of the model is not very cheap , so that makes teaching resources are tight, but also reach a very good teaching. Radar semi-physical simulation system is teaching and training can solve the above problems, for a presentation by non-operating part of the model instead, and some of the specific operation by the computer instead. Used by the host analog signal received by the receiver, and display the data passed to the client, then the client displays to the students by the students to some of the simulation operation. Using both the host teacher can monitor the operation to facilitate teaching students.

Radar-loop simulation teaching and training systems using C/S architecture, which is composed of two parts, namely, the client application and server program. Both procedures can be called the front and back, respectively, the program. The machine running the database server program, known as application servers, once the server program is started, you always wait for a response sent to client requests; client program running on the user's own computer, corresponding to the server computer, client computers can be called. When the need for any data in the database operation, the client will automatically find the server program to its request, the server process to make a response in accordance with predetermined rules, return the result.

Radar-loop simulation teaching and training system to take real-time by the host according to time data from the database necessary, and calculate the real-time image display data needed. Then sends the data through the network to the client program, the client receives data in accordance with real-time image displayed on the terminal. When the need for data in the database query operation, the client program will automatically find the server program to its request, the server process in accordance with predetermined rules to make a response back to the client application needs the data results.

\section{SYSTEM DESIGN AND IMPLEMENTATION}

Radar-loop simulation teaching and training system has three main components: demonstration model, the main console (host), the client.

\section{A. Model presentation}

Demonstration model is to demonstrate the radar outside the main operation of the operation and demonstration, for focusing on the teacher in class. Radar demonstration model of the external part of the structure can have, radar launch demonstration, the radar antenna rotation speed control presentation functions. This keeps the students through a more intuitive presentation of the model have an intuitive understanding of the radar.

Main antenna from the antenna frame, shaped reflector network, feed, feed bracket, antenna turntable, antenna mounts, gear position, orientation drive motor and other components.

Radar antenna reflector is $10 \mathrm{~m}$ (length) $\times 5 \mathrm{~m}$ (high). Simulation model is 20 times smaller (or 10-fold) production, namely the ratio of $1: 20$ (or $1: 10$ ).

In addition to the radar antenna reduced by physical simulation model, it also has the following features:

(1) the antenna can work forward and reverse; (2) around the antenna speed $10 \mathrm{r} / \mathrm{min}$ (select the appropriate speed motor and gear ratio to achieve); (3) antennas to sweep fan work, sweep the need to increase the low voltage power supply fan, the use of time (or count) control circuit, the antenna relay control by inversion, form a fan antenna sweep mode; (4) azimuth drive motor, the azimuth drive motor single-phase low-speed motor and gear reducer to achieve antenna drive again.

A Radar cabins to 4-meter standard cabins. Shelter in front of the studio work is followed by the transmitter room and studio by the three cabinets. Control cabinet (including industrial computer, monitor display, monitoring console, etc.); terminal cabinet (including self-recording stations, the terminal screen, taking console, etc.); integrated cabinet 
(including the receiver extension, signal processing extensions, etc.). Transmitter room is mainly by the launch cabinets.

B radar cabins to 4-meter standard cabin. Simulation models produced by 10 -fold reduced, is the ratio of 1:10.

\section{$B$. radar simulation training system teaching the main console}

Plus bone aluminum cabinet and the use of advanced mining technology into glue. 19-inch LCD screens, and with the console, keyboard, mouse, console the bottom of the server host.

(1) Radar, open, shut down operations; (2) Antenna start and stop operation; (3) antenna rotation speed control; (4) Other operating functions.

Host software to complete the main operating functions is: the analog signal, the signal set, sending the signal data, monitoring client, database operations.

Mainly analog signal receiver receives signals through a computer simulation on the data displayed on the terminal. This allows students to see the computer screen with the radar display terminal to see almost the same interface. Signal simulation includes simulation of terrain, clutter simulation, flight simulation of the target route, flight simulation target.

Signal set is necessary to set the analog signal, you can work separately on the radar antenna parameters, terrain types, the type of clutter, the target flight data and flight simulation of the target track data to establish a database, set the host analog signal, respectively, to extract records in the database selection be combined to generate flexible client interface to the actual data. For the signal set includes two settings: one is only in the initialization can be set during operation can not be set, such as terrain conditions; a can be set at any time, such as aircraft flight, radar antenna speed.

Transmit the data signal is mainly produced in the host analog signal data sent over the network to each client, the client receives data on the data can be converted into images displayed on the terminal computer display.

Management operations mainly for teachers and students as well as manage client information, and when the system is running real-time monitoring client status, such as the client is online and other information. Host information management includes staff management, student information management, client information management, client on-line monitoring of four parts.

Since many signal simulation need to provide fixed parameters, some of the waveform may be by sampling the data, while in operation, such as teacher's user name and password storage and teaching modules to use the database, such as test database, so it is necessary to use the database . Database information mainly includes three parts: the user-related information, such as: teacher log host user name and password; analog signal necessary parameters such as size of the aircraft heading; teaching module related data, such as the test database, etc. .

\section{Client}

Host sends data over the network to the client, the client only need to display data received in the simulated radar terminal display screen for operation with trainees. Clients can achieve a goal of operating information display, real-time image display, and target operating and teaching using four parts.

Clients receive the host from the network based on simulated radar echo signal data through the graphics-display screen on the client, to simulate the radar terminal display.

Client acquires from hosts on the network objectives of the campaign information, and the specific operation according to the students show up. Target information targeted speed, target distance, target angle, target track and other information.

\section{Target Operating}

Students can be simulated on the client terminal radar operations, but because some settings can only be set by the host, the client can not set up operations, such as antenna rotation speed setting, the transmitter switch settings, turn the antenna switch. Operation of the specific is content of the target object detection, moving target tracking, target track display.

\section{E. Teaching Use}

Teaching is mainly used for teaching module applications, such as trainees through the principle of client self-radar, the questions generated by the test database for testing and other operations.

\section{CONCLUSIONS}

1) The system uses the $C / S$ architecture, scalability, as part of the system's main operations and database installed on the host, so the client can add a lot to reduce the workload.

2) The system's main data processing from the host processing, the client only for image display processing, so the client can guarantee consistent display of graphics to facilitate teaching.

3) Visual effect of the system, because it is an analog signal through the computer, so you can simulate a variety of environments in the terminal display.

4) Availability of the system is good, provided a number of alternative air routes, teachers can be set by the host, and participants can simulate real-radar operations.

Radar-loop simulation teaching and training system is simple, easy to use, affordable, easy maintenance, high efficiency of teaching, in the general promotion of the institutions, but also particularly suitable for combat forces, in particular radar observation and teaching soldiers to use. At the same time, the system will not become obsolete based on the principle, into a series of related products, development and production batches.

\section{REFERENCES}

[1] Liu YanBing, Jin Guang. Development Status of Hardware-in-loop Simulation $[\mathrm{J}]$. Optics,Mechanics \& Electronics Information, 2003(1):27-32 
[2] Jiang Zhen, Shao Dingrong, Xiong Huagang, et al. Research on the Reliability Model of Real-time Network Systems [J]. Acta Aeronautica Et Astronautica Sinica, 2004,25(3): 275-278

[3] Wang Jian. Design and Implementation of Hardware-in-the-loop Simulation of Radar System [D]. Xidian University, 2009.

[4] Ding Lufei Geng Fulu. Radar Principles [M]. Xian: Xidian University Publishing House, 2002.
[5] Xiang Jingcheng, Zhang Mingyou. Radar System [M]. Beijing: Publishing House of Electronics Industry, 2001.

[6] Ni Guoqi, Li Runian. Radar Principles and Application of Simulation Training [M]. Beijing: PLA Publishing House, 2002.

[7] Pan Zhiming, Yin Chengzhi. Research on Radar Target Simulation System [J]. Electronic Engineer, 2003, 29(7):56-58 